\title{
Androgen receptor expression in circulating tumour cells from castration-resistant prostate cancer patients treated with novel endocrine agents
}

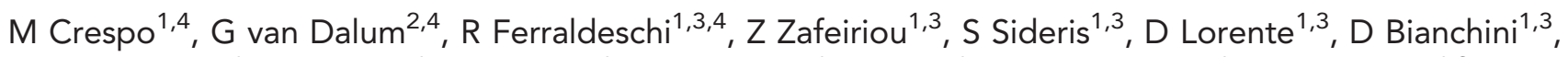
D N Rodrigues ${ }^{1}$, R Riisnaes ${ }^{1}$, S Miranda ${ }^{1}$, I Figueiredo ${ }^{1}$, P Flohr ${ }^{1}$, K Nowakowska ${ }^{1}$, J S de Bono ${ }^{1,3}$, L W M M Terstappen ${ }^{2}$ and G Attard ${ }^{\star, 1,3}$

${ }^{1}$ Section of Medicine, The Institute of Cancer Research, Sutton, Surrey, SM2 5NG, UK; ${ }^{2}$ Department of Medical Cell BioPhysics, MIRA Institute, University of Twente, 7522ND, Enschede, The Netherlands and ${ }^{3}$ Prostate Cancer Targeted Therapy Group and Drug Development Unit, The Royal Marsden NHS Foundation Trust, Sutton, Surrey, SM2 5NG, UK

Background: Abiraterone and enzalutamide are novel endocrine treatments that abrogate androgen receptor (AR) signalling in castration-resistant prostate cancer (CRPC). Here, we developed a circulating tumour cells (CTCs)-based assay to evaluate AR expression in real-time in CRPC and investigated nuclear AR expression in CTCs in patients treated with enzalutamide and abiraterone.

Methods: CTCs were captured and characterised using the CellSearch system. An automated algorithm to identify CTCs and quantify AR expression was employed. The primary aim was to evaluate the association between CTC AR expression and prior treatment with abiraterone or enzalutamide.

Results: AR expression in CTCs was evaluated in 94 samples from 48 metastatic CRPC patients. We observed large intra-patient heterogeneity of AR expression in CTCs. Prior exposure to abiraterone or enzalutamide was not associated with a change in CTCs AR expression (median intensity and distribution of AR-positive classes). In support of this, we also confirmed maintained nuclear AR expression in tissue samples collected after progression on abiraterone. AR staining also identified additional AR-positive CD45-negative circulating cells that were CK-negative/weak and therefore missed using standard protocols. The number of these events correlated with traditional CTCs and was associated with worse outcome on univariate analysis.

Conclusions: We developed a non-invasive method to monitor AR nuclear expression in CTCs. Our studies confirm nuclear AR expression in CRPC patients progressing on novel endocrine treatments. Owing to the significant heterogeneity of AR expression in CTCs, studies in larger cohorts of patients are required to identify associations with outcome.

Castration-resistant prostate cancer (CRPC) is the third cause of cancer-related death among men, accounting for a predicted 10.19 deaths per 100.000 men in 2014 in Europe (Malvezzi et al, 2014). Although targeting of the androgen receptor (AR) with abiraterone or enzalutamide improves survival in CRPC (de Bono et al, 2011; Scher et al, 2012), resistance invariably develops and the response rate to sequential treatment appears lower when one agent is used after the other (Loriot et al, 2013; Bianchini et al, 2014).

\footnotetext{
*Correspondence: Dr G Attard; E-mail: Gerhardt.attard@icr.ac.uk

${ }^{4}$ These authors contributed equally to this work.
}

Received 18 October 2014; revised 19 January 2015; accepted 27 January 2015; published online 26 February 2015 (C) 2015 Cancer Research UK. All rights reserved 0007 - 0920/15 
Nonetheless, a rise in serum prostate-specific antigen (PSA) is observed in the majority of patients progressing on either treatment, suggesting reactivation of the AR signalling pathway and maintained PSA expression. In support of this, PSA expression together with downregulation of PSMA has been demonstrated in circulating tumour cells (CTCs) from patients progressing on abiraterone (Miyamoto et al, 2012). Although expression of PSA is predominantly under the control of the AR in early prostate cancer, recent studies suggest that other steroid receptors, such as the glucocorticoid receptor share DNA-binding sites with the AR, can induce expression of PSA and other key AR-regulated genes and in doing so, mediate resistance (Arora et al, 2013; Sahu et al, 2013). In addition, the widespread use of AR-targeted therapies may produce the emergence of prostate cancer that no longer depends on AR signalling with neuroendocrine features (Beltran et al, 2012). A major question currently is whether AR-based progression is a predominant mechanism mediating resistance or whether other mechanisms, including bypass of AR and an ARindependent prostate cancer, are more common in CRPC progression.

Although we and others have established tumour biopsy programs for CRPC patients, a significant proportion of patients do not have disease that is amenable to biopsy and not all biopsies contain cancer. Moreover, single tumour biopsies may not be representative of all metastases. CTCs can be isolated from blood in a non-invasive approach, and serve as a 'liquid biopsy' that might provide the opportunity to non-invasively and repeatedly sample representative tumour cells, and thus provide information concerning molecular characteristics of tumour, tumour heterogeneity and changes that occur with treatment in real-time. Two studies highlighted the potential utility of assessing dynamic changes in AR in CTCs in monitoring CRPC (Darshan et al, 2011; Miyamoto et al, 2012).

Various methods have been developed for the identification and isolation of CTCs in blood, mostly employing enrichment strategies based on the physical properties or size, or positive/ negative selection using cell-surface antigens or other biological properties of these cells (Attard and de Bono, 2011). To date, the CellSearch system is the only Food and Drug Administration (FDA)-approved platform for the capture, analysis and enumeration of CTCs. The CellSearch system, in addition to enumeration of CTCs, offers the advantage for additional characterisation with user-defined markers of interest, by adding an antibody directly conjugated to fluorescein isothiocyanate (FITC) and further image capture on the fourth fluorescence (FITC) channel. This approach has previously been used to individually study various cell surface markers, including IGF-1R, Her-2 and EGFR (de Bono et al, 2007; Punnoose et al, 2012; Ligthart et al, 2013; Liu et al, 2013). However, to detect nuclear markers, an additional 'off-line' permeabilisation step and staining of CTCs has previously been described as a necessary step to detect nuclear expression of proteins such as phosphorylated $\mathrm{H} 2 \mathrm{AX}(\gamma \mathrm{H} 2 \mathrm{AX})$ (Wang et al, 2010). In this study, we aimed to develop an online CTC-based assay on the CellSearch system for real-time quantitation of nuclear AR expression in CRPC patients, and investigate the association between nuclear AR expression and treatment with novel AR-targeting agents, enzalutamide and abiraterone.

\section{MATERIALS AND METHODS}

Patients. Peripheral blood $(7.5 \mathrm{ml})$ was collected from metastatic CRPC patients with histologically confirmed prostate cancer starting or progressing on abiraterone or enzalutamide and from healthy volunteers. All patients and healthy donors gave their written informed consent and were enrolled on institutional protocols approved by the Royal Marsden NHS Foundation Trust (London, UK) Ethics Review Committees (Research Ethics Committee (REC) 04/Q0801/60).

Cell lines and drug treatment. Prostate cancer cell lines 22Rv1 (\#CRL-2505), LNCaP (\#CRL-1740), VCaP (\#CRL-2876), PC3 (\#CRL-1345) and DU145 (\#HTB-81) were obtained from the American Type Culture Collection (ATCC) and cultured according to ATCC recommendations. Enzalutamide was synthesised using the publicly available chemical structure and checked by mass spectrometry.

AR immunofluorescence (IF) staining on CTCs and manual CTCs enumeration. Peripheral blood was collected into CellSave preservative tubes (\#7900005, Janssen Diagnostics, LLC, Raritan, NJ, USA) and processed within $96 \mathrm{~h}$. The CellSearch system (Janssen Diagnostics, LLC) was used for the enumeration of CTCs. This technology relies on the expression of epithelial cellular adhesion molecules by epithelial cells and the isolation of these cells by immunomagnetic capture using anti-epithelial cellular adhesion molecule antibodies. The enriched cells were stained by IF using antibodies specific for Cytokeratin (keratin 8, 18 and 19) conjugated to Phycoerythrin (CK-PE), anti-CD45 conjugated to Allophycocyanin (CD45-APC) and the nucleic acid dye $4^{\prime}$, 6-diamidino-2-phenylindole (DAPI). A user-defined assay utilising the CellTracks Autoprep system for the detection of AR by IF on single CTCs was performed with an AlexaFluor 488-conjugated rabbit monoclonal antibody (clone D6F11, \#7395, Cell Signaling Technology, Danvers, MA, USA) directed against the aminoterminus domain of the AR. The antibody was used at a concentration of $2.5 \mu \mathrm{g} \mathrm{ml}^{-1}(1: 10)$ diluted in PBS and a permeabilisation solution to improve intracellular/nuclear staining with a concentration of $5 \times$ Perm/Wash Buffer (BD Biosciences, San Jose, CA, USA). For one sample, we mixed $45 \mu \mathrm{l}$ of AR antibody from a $25 \mu \mathrm{g} \mathrm{ml}^{-1}$ stock solution to $225 \mu \mathrm{l}$ of a stock solution of $10 \times$ Perm/Wash Buffer and $180 \mu \mathrm{l}$ of PBS for a final volume of $450 \mu \mathrm{l}$. The antibody mixed with Perm/Wash buffer and PBS solution was then added to the CellSearch reagent cup and placed in the position one of the carrier reagent tray of the CellTracks Autoprep system. Images of stained events were captured on the CellTracks Analyzer II (Janssen Diagnostics, LLC) and manually examined to determine the presence of CTCs and number of AR-positive CTCs. An event was classified as a manual CTC (mCTC) if the features were consistent with an epithelial tumour cell as previously described (Allard et al, 2004). The number of mCTC is presented per $7.5 \mathrm{ml}$ of blood. All images on the fourth channel (FITC) were captured using an integration time of $0.8 \mathrm{~s}$.

Automated CTC enumeration and nuclear AR quantitation. The exported image files from the CellTracks Analyzer II were reanalyzed using an automated algorithm developed in Matlab (Matworks, Natick, MA, USA) as previously described (Ligthart et al, 2011, 2013). This algorithm was modified to measure nuclear AR expression (Figure 1A). Briefly, this algorithm evaluates all CKPE-positive events above a threshold determined per cartridge. An event becomes an automatically detected CTC (aCTC) when it meets the following attributes: size range between 75 and 500 pixels, CK-PE standard deviation above 50, a DAPI peak value of at least 170 arbitrary units (a.u.) and a CD45-APC maximum value of less than 60 a.u. If an event had a standard deviation for CK-PE below 50, it was classified CK-negative/weak. A cartridge-wide threshold was also determined for DAPI. The average AR expression inside both the nuclear mask and the cytokeratin-based mask was calculated. The AR-FITC was corrected by subtracting the surrounding background. Automated classification for whether a CTC was positive or negative for AR was based on the threshold set using AR-negative prostate cell lines (25 a.u.). 
A

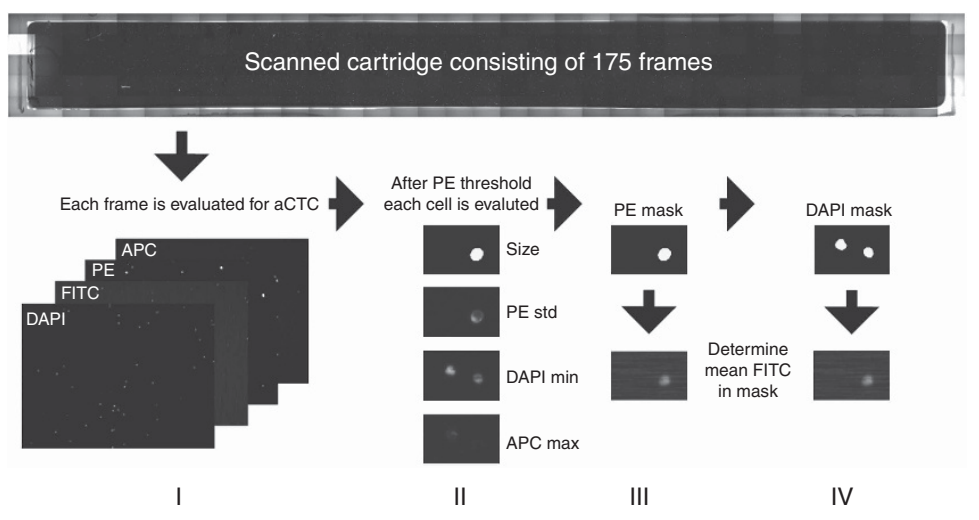

C

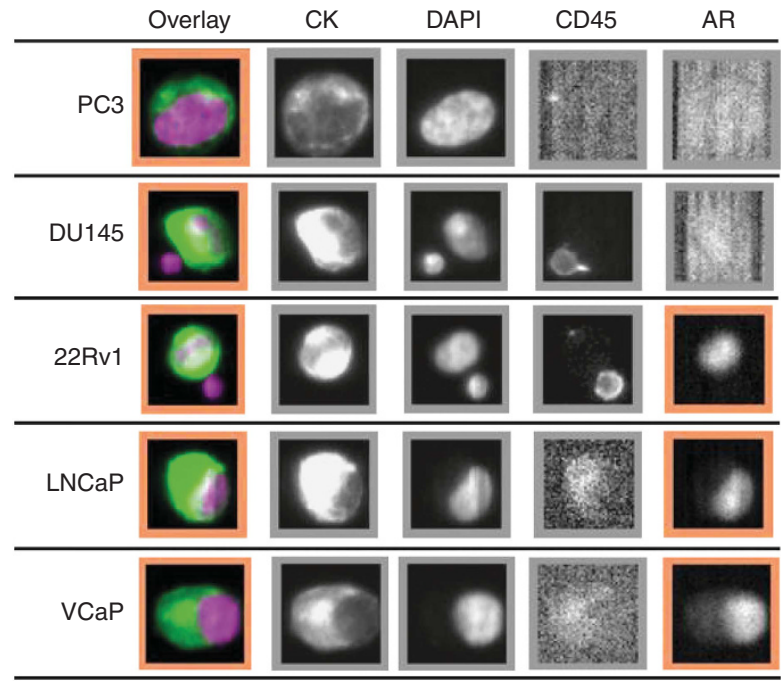

B
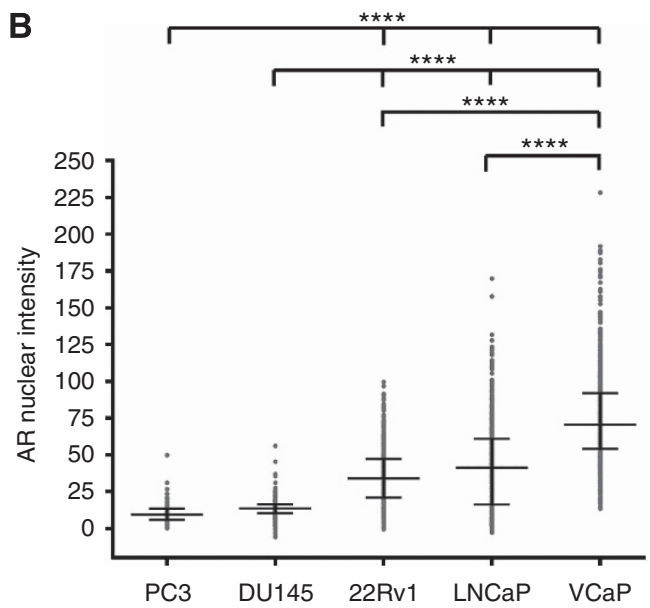

D

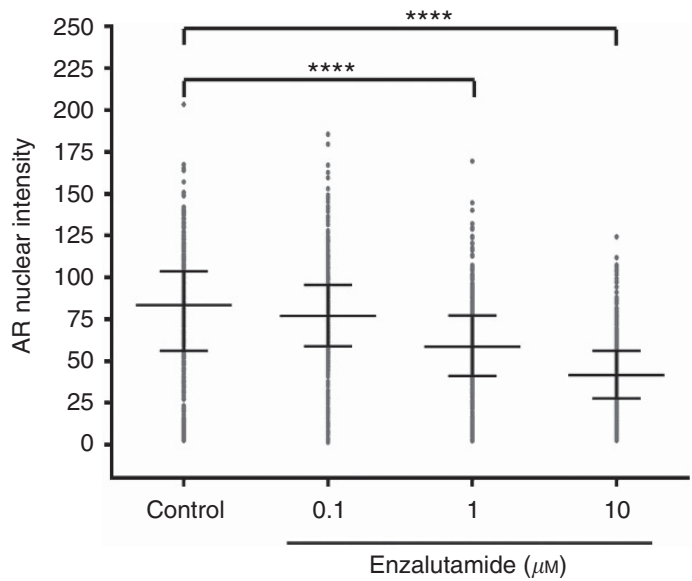

Figure 1. Assay development for the AR marker using the CellSearch system. (A) Mean AR intensity was determined by automated image analysis. Each frame was evaluated for CTC using a global CK-PE threshold (I). After classification (II), the mean AR-FITC intensity was determined inside the Cytoplasm (III) and Nucleus (IV). (B) Prostate cancer cell lines were spiked in healthy volunteer (HV) blood and detected on the CellSearch platform. Median nuclear AR intensity of individual cells \pm interquartile range (IQR) is plotted. AR nuclear intensity was determined using the automated algorithm. ${ }^{* \star * *} P<0.001$, One-way ANOVA followed by Dunett's multiple comparison test. (C) Representative images of AR expression in five prostate cancer cell lines detected on the CellSearch platform. PC3 and DU145 cells were used as negative controls and displayed absence of AR expression. 22Rv1, LNCaP and VCaP were used as positive controls. (D) LNCaP cells were grown in RPMI supplemented with $10 \%$ FBS and treated with indicated concentrations of enzalutamide or vehicle (DMSO $0.2 \%$ ) for $24 \mathrm{~h}$ before spiking in HV blood. Cells were then isolated and detected on the CellSearch platform. AR nuclear intensity was evaluated using the automated algorithm. Median $\pm I Q R$ is showed. ${ }^{\star * \star \star} P<0.001$, One-way ANOVA followed by Dunett's multiple comparison test.

Fluorescent in situ hybridisation (FISH). FISH was performed on CTCs as previously described (Attard et al, 2009b) with probes covering regions at the centromere of the X chromosome (APClabelled) and the $A R$ gene (FITC-labelled). Fluorescent in situ hybridisation signals in individual CTCs were determined to assess $A R$ gene status. $A R$ amplification was considered to be present when the $A R$ gene to $\mathrm{X}$ chromosome ratio was greater than 1.5 . Leukocytes were used as internal controls.

Multiplex IF on tissue. Multiplex IF staining was performed on $4 \mu \mathrm{m}$ sections from formalin-fixed paraffin-embedded tissue using antibodies against CD45 (\#M070129-2, 1:200, clones 2B11+ PD7/26, Dako UK Ltd, Ely, Cambridgeshire, UK) detected with a goat anti-mouse antibody-conjugated to AlexaFluor 555 (\#A21422, $1: 200$, Life Technologies, Paisley, UK); Pan-cytokeratin-conjugated to AlexaFluor 647 (\#4528, 1:200, clone C11, Cell Signaling) and AR-conjugated to AlexaFluor 488 (\#7395, 1:50, clone D6F11, Cell Signaling). Slides were imaged with a multispectral automated fluorescence microscope (Vectra, PerkinElmer, Seer Green, Beaconsfield, Buckinghamshire, UK) and AR nuclear intensity analysed using inForm v.2.0 (PerkinElmer).

Statistical analysis. The primary aim was to evaluate the association between CTC AR expression and prior treatment with abiraterone or enzalutamide. Secondary aims included evaluation of CTC AR expression and changes in CTC AR expression and clinical response as defined by PCWG2 criteria (Scher et al, 2008). Independent $t$-tests, and Pearson's Chi-Square were used to compare continuous and categorical variables. All tests were twosided and a $P$-value of 0.05 or less was considered statistically significant. Spearman's rank correlation was used to evaluate the degree of correlation between two continuous variables. Survival was measured from the date of first blood draw to the date of last contact or death from any cause. The Kaplan-Meier product-limit method was used to estimate the median overall survival. The survival rates were compared using the log-rank test. Univariate 
and multivariate analysis of the independent factors for overall survival was performed using the Cox proportional hazard regression model with a 95\% confidence interval. Descriptive statistics and survival analyses were performed using IBM SPSS Statistics v.22 (IBM, Portsmouth, Hampshire, UK) and GraphPad Prism v.6.04 (GraphPad Software, Inc., La Jolla, CA, USA).

\section{RESULTS}

Evaluation of AR expression in circulating prostate cancer cells. For CTC isolation and characterisation, we used the CellSearch system. The platform allows introduction of additional staining with an antibody conjugated to a fourth fluorophore. In this study, we aimed to develop an assay to detect AR expression in CTCs by introducing an anti-AR antibody directly conjugated with AlexaFluor 488 (AR-AF488) to the immunostaining step on the CellSearch platform. The AR-AF488 antibody binds the aminoterminus of the receptor and therefore it can detect both full length and the constitutively active truncated AR splice variant (e.g., ARV7). We first established the optimal AR-AF488 antibody concentration, by testing a range of concentrations on $\mathrm{VCaP}$ (AR-positive) and PC3 (AR-negative) prostate cancer cells, first plated directly onto slides (Supplementary Figure 1) and subsequently spiked into blood and captured on CellSearch. We then confirmed that cells need to be maintained in a permeabilised state during incubation with AR-AF488 in order to achieve the best intracellular/nuclear staining (Supplementary Tables 1 and 2). Our assay had the advantage that captured cells were permeabilised and stained for AR, CK, CD45 and DAPI in one step without removal of cartridges from the CellSearch system. We were able to distinguish differential levels of nuclear AR in a broader panel of prostate cancer cell lines and decreases in expression of nuclear AR in LNCaP cells treated with the AR antagonist enzalutamide (Figure 1B-D). We then compared our assay with the standard CellSearch protocol and found no significant differences in CTC numbers in two contemporaneously collected samples from 14 patients $(P=0.5$, Wilcoxon's signed-rank test) (Supplementary Table 3). Only in 1 of the 14 samples examined, the classification changed from favourable (4 CTCs) to unfavourable (8 CTCs) when the AR antibody was added. Next, to determine assay variability for CTC detection and AR expression, we compared replicate samples collected at the same time from three patients (Supplementary Tables 4 and 5). Minimal intra-run, intra-day and inter-day variability was observed.

AR expression in CTCs from CRPC patients. AR expression in CTCs was evaluated in 94 samples from 48 metastatic CRPC patients. Details of patients' characteristics are summarised in Table 1. The standard CellSearch operator-scored CTC (mCTC) count ranged from 0 to 4268 (median 8, mean 133.8) with 1 or more CTC identified in $80 / 94$ samples (85\%). In addition to enumerating CTCs, we also manually scored CTCs as AR-positive or -negative. However, operator quantitation of IF staining could be subject to bias and could be especially inaccurate on CellSearch owing to artificial post-capture enhancement of images. We therefore adapted our previously published algorithm (Ligthart et al, 2011) to allow automated identification and quantitation of nuclear AR in CTCs. We subjected unprocessed captured data to automated identification and quantitation of CK, CD45 and AR staining intensity. The correlation between the numbers of events categorised as CTCs in each sample by standard operator (mCTC) and automated assessments (aCTC) was good with an $R^{2}$ of 0.94 (Figure 2A). As previously reported in other patient cohorts (Ligthart et al, 2011), an unfavourable aCTC count ( $\geq 4 \mathrm{CTC} /$ $7.5 \mathrm{ml}$ ) was significantly associated with worse survival in our 48
Table 1. Patients' demographics and clinical characteristics

\begin{tabular}{|c|c|}
\hline & $N=48$ \\
\hline \multicolumn{2}{|l|}{ Age, years } \\
\hline Median, range & $68(40-82)$ \\
\hline \multicolumn{2}{|l|}{ Sites of metastases } \\
\hline Bone & $43(90 \%)$ \\
\hline Nodal & 20 (42\%) \\
\hline Visceral & $16(33 \%)$ \\
\hline \multicolumn{2}{|l|}{ ECOG PS } \\
\hline 0 & $8(17 \%)$ \\
\hline 1 & 37 (77\%) \\
\hline 2 & $3(6 \%)$ \\
\hline \multicolumn{2}{|l|}{ Haemoglobin, $\mathrm{g} \mathrm{dl}^{-1}$} \\
\hline Median, range & $12.2(8.3-15.5)$ \\
\hline \multicolumn{2}{|c|}{ Lactate dehydrogenase, $\mathrm{IUI}^{-1}$} \\
\hline Median, range & $174(112-678)$ \\
\hline \multicolumn{2}{|l|}{ Albumin, $\mathrm{g} \mathrm{dl}^{-1}$} \\
\hline Median, range & $35(24-42)$ \\
\hline \multicolumn{2}{|c|}{ Previous treatment for CRPC } \\
\hline Docetaxel & $33(69 \%)$ \\
\hline Abiraterone & 22 (46\%) \\
\hline Enzalutamide & $4(8 \%)$ \\
\hline Cabazitaxel & $9(18 \%)$ \\
\hline Investigational agents & $7(15 \%)$ \\
\hline
\end{tabular}

patients (HR 3.2 95\% confidence interval 1.3-6.5 Log-Rank $P=0.01$ ) (Supplementary Figure 2). Of 11636 aCTCs, 4223 (36\%) were AR-negative by automated algorithm. Mean cell AR intensity (overlapping with the CK mask) and nuclear AR intensity (overlapping with the DAPI mask) for the 7413 AR-positive aCTC were calculated. We observed a high correlation between the classification of manually scored and automated CTC as ARpositive but poor correlation for intensity of AR staining. This was in keeping with our expectation given post-capturing processing and enhancement of images presented on CellSearch, which occurs prior to operator review. We confirmed that median AR intensity and percentage of AR-positive aCTC was not influenced by total aCTC number or number of white blood cells (Supplementary Figure $3 \mathrm{~A}$ and $\mathrm{B}$ ). The median AR intensity of white blood cells was below the set threshold to define AR positivity (Median 4.8; Minimum 0.4-Maximum 13.1). Interestingly, when reviewing images, we observed a correlation between non-intact CTCs (with pycnotic and/or fragmented nuclei) and weak/absent AR staining (Figure 2B), defined by distinct features shown in Figure 2C. This suggested that AR negativity in CTCs may be at least in part related to in vivo or ex vivo artefacts related to loss of cellular integrity.

CTC AR nuclear expression in abiraterone- or enzalutamideresistant CRPC. We then examined AR expression in aCTCs from CRPC patients according to prior exposure to abiraterone or enzalutamide therapy. We selected samples with at least four aCTCs and classified every aCTCs as AR-negative or AR-positive and then every one of the AR-positive aCTC into one of four quartiles (Figure 2D). We observed inter-patient and intra-patient heterogeneity in expression of AR in aCTCs (Figure 3A). Overall, we did not find significant changes in AR expression (median intensity and distribution of AR-positive classes) between aCTC from abiraterone and enzalutamide-naïve patients $(n=11)$ and patients who had progressed on abiraterone or enzalutamide but continued $(n=9)$ or discontinued $(n=12)$ treatment (Figure $3 \mathrm{~B})$. Median nuclear AR intensity was 17.8 (IQR 13.9-50.6) in the 

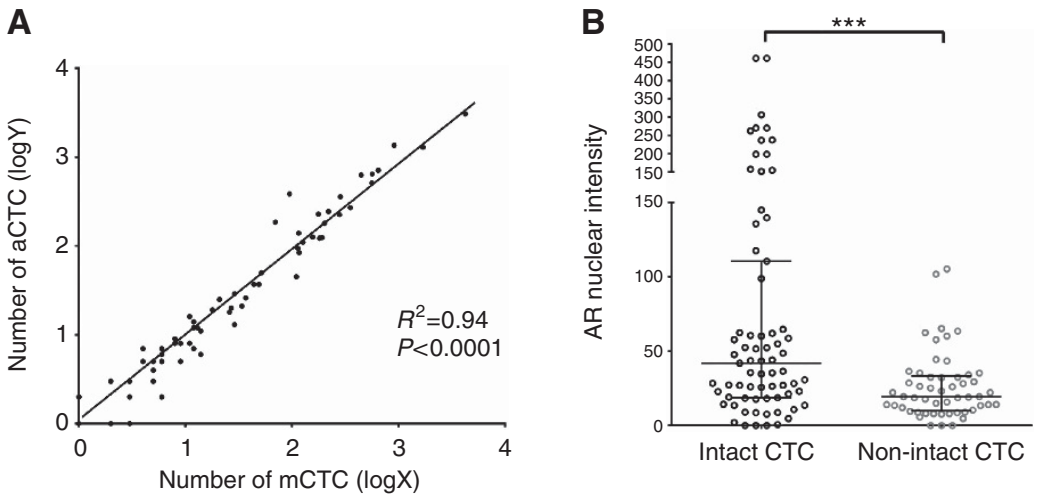

C
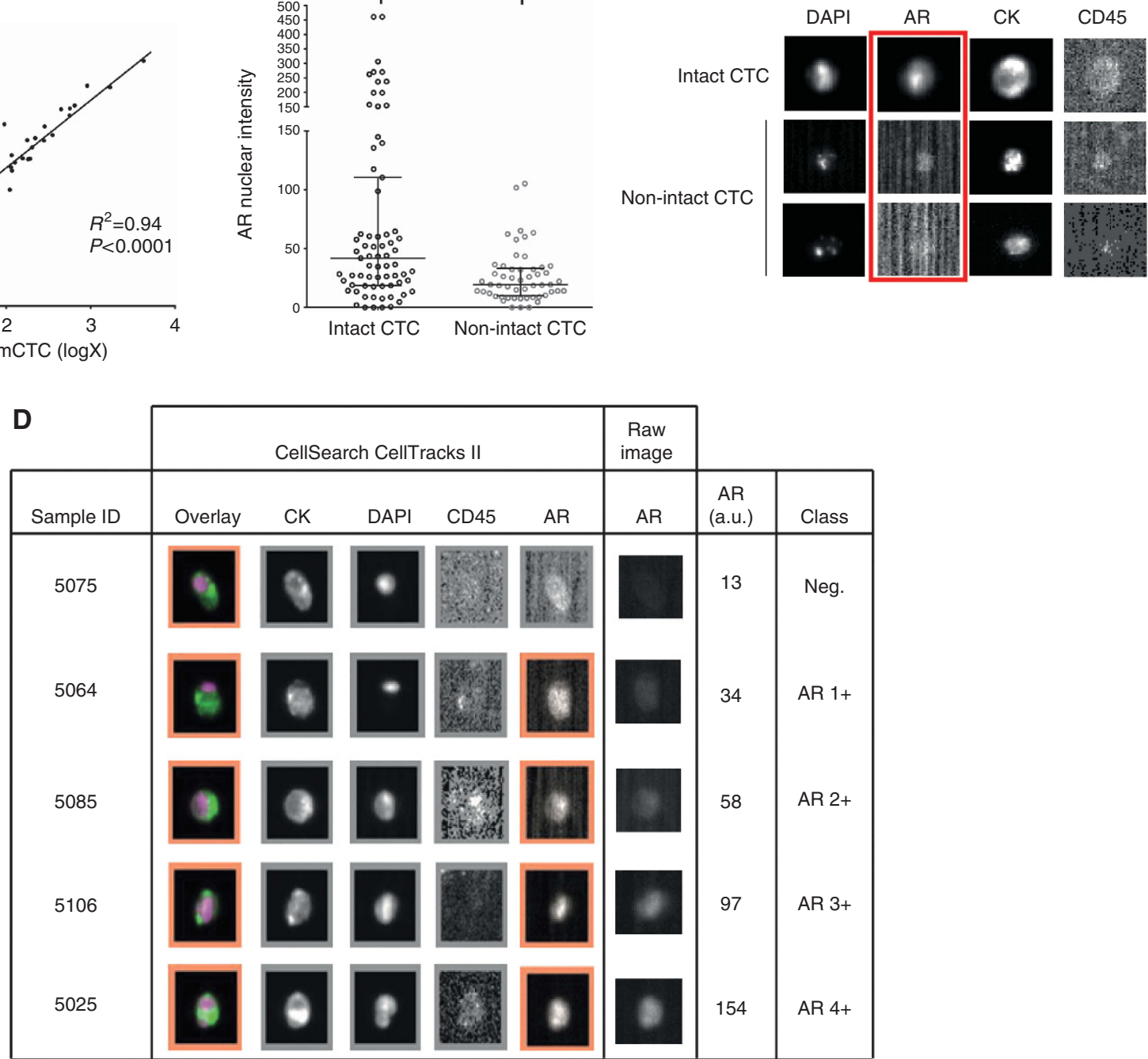

Figure 2. CTC enumeration and AR quantitation of individual CTCs in clinical samples. (A) Linear correlation between manual (m)CTC counts in each sample as evaluated by standard operator ( $\mathrm{mCTC}$ ) and the unbiased automated (aCTC) counts as determined by the computer algorithm. (B) Comparison of AR intensity in CTCs with pycnotic and/or fragmented nuclei (non-intact) and in intact CTCs. Median AR intensity of individual cells $\pm I Q R$ is shown. Mann Whitney test, $P=0.0002$. (C) Representative images of one intact and two non-intact CTCs. Non-intact CTCs can be seen with distinct features such as cell shrinkage and nuclear fragmentation and absent AR expression on the FITC channel (red rectangle). (D) Representative images showing AR expression of individual CTC from five different CRPC patients divided into four quartiles depending on the nuclear AR intensity (AR1 + , AR2 +, AR3 + and AR4 +). CTCs with AR expression below the threshold ( $<25$ a.u.) were classified as negative. Raw TIFF files were used for the purpose of AR quantitation with the computer algorithm.

abiraterone- and enzalutamide-naïve patients, 18.7 (IQR 8.0-35.1) in patients who had progressed on abiraterone or enzalutamide but still receiving treatment at the time of the blood draw, and 23.3 (IQR 10.9-35.8) in patients who had previously received abiraterone or enzalutamide and discontinued treatment owing to progression. Median percentage of AR-positive CTCs was 29\% (IQR 10-71), 35\% (5-54) and 44\% (IQR 20-65), respectively. The difference was not significant (Supplementary Figures $4 \mathrm{~A}$ and $\mathrm{B}$ ).

We also investigated intra-patient changes in AR expression in eight patients with sequential samples collected before starting treatment and at progression. In five patients, there was an increase in median nuclear $\mathrm{AR}$ expression in aCTC at progression compared with pre-treatment (Figure 3C). Overall, these data suggest that AR nuclear expression is maintained or even increased in CTC at progression on abiraterone and enzalutamide in a proportion of patients. We did not identify a shift to AR-negative CTC in our cohort of patients. To support these CTC data, we also performed AR IF on fresh tumour tissue biopsies from 13 CRPC patients progressing on abiraterone and still on-treatment and confirmed maintained nuclear AR expression (Figure 3D).

Detection of AR-positive and CK weak/negative circulating cells. Automated analysis of epithelial cellular adhesion moleculepositive events captured on CellSearch with additional AR staining identified a sub-class of AR-positive, CD45-negative, intact, nucleated circulating cells that had negative or weak CK (CK-) expression. CK-AR + CD45- nucleated cells counts ranged from 0 to 286 (median 2; mean 21) in CRPC patients and significantly correlated with traditional CK-positive aCTC $\left(\mathrm{R}^{2} 0.72 ; P<0.0001\right)$ (Figure 4A). More than four CK-AR + CD45- events were found in 18/48 (37.5\%) CRPC patients and were not observed in male healthy volunteers or other tumour types (Figure 4B). We detected $A R$ amplification or $A R$ copy number/X chromosome gain in three of these patients supporting a malignant origin of these CK weak/ negative circulating cells (Figure $4 \mathrm{C}$ ). The total number of $\mathrm{CK}$ 
A AR expression measured in individual CTC

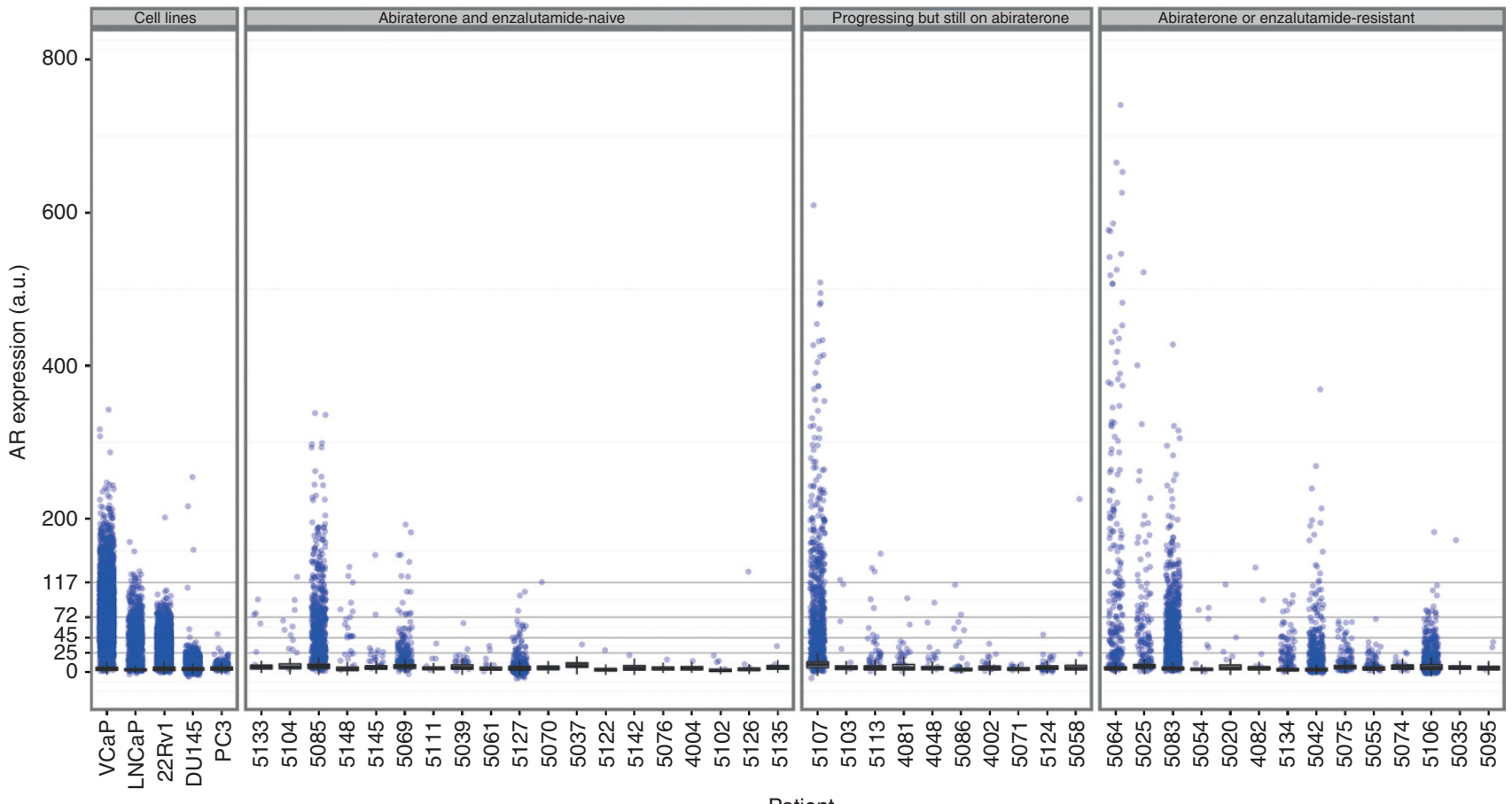

Patient
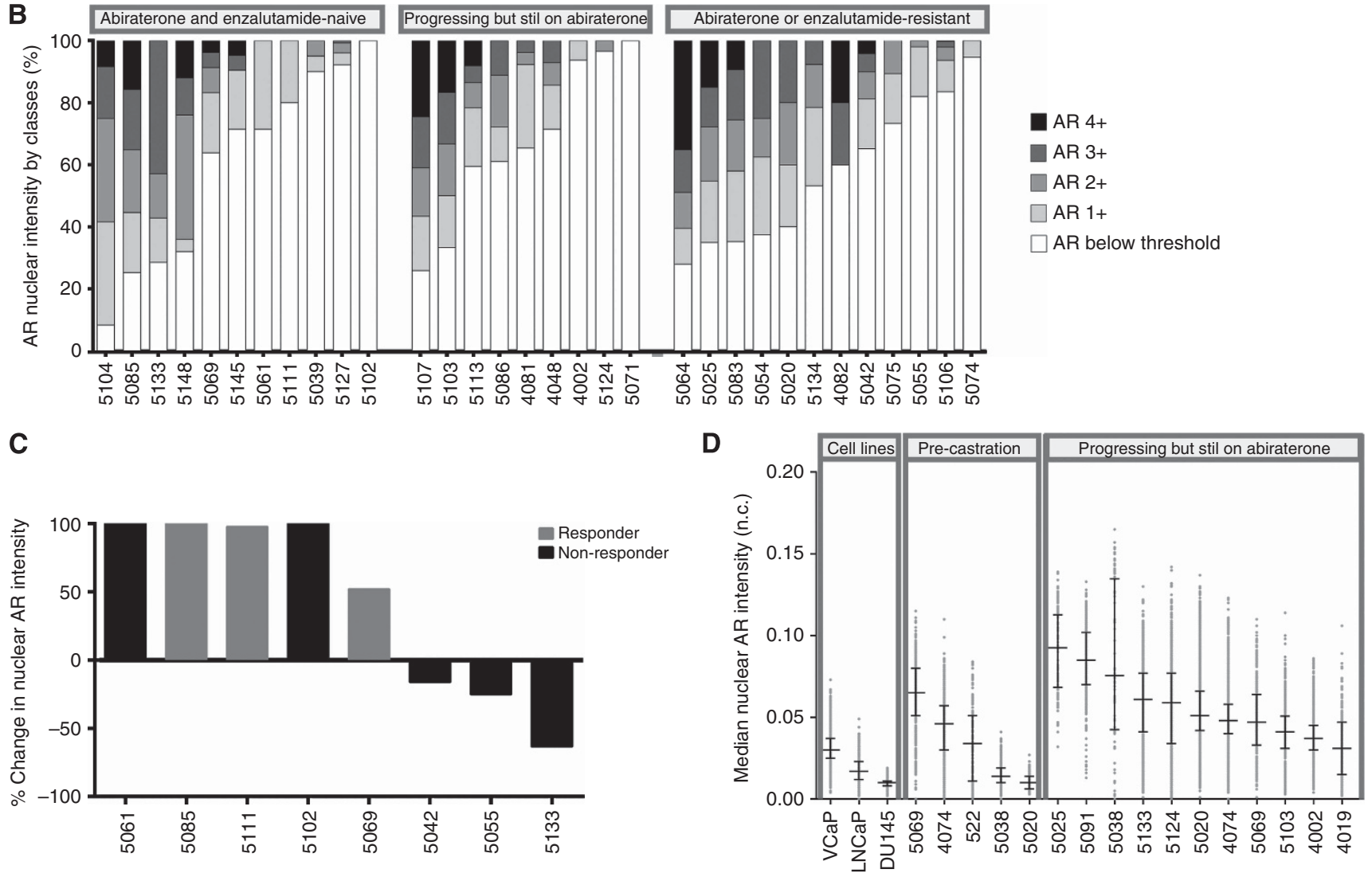

Figure 3. CTC AR nuclear expression in abiraterone- or enzalutamide-resistant CRPC patients. (A) AR intensity in individual CTC in samples from abiraterone- and enzalutamide-naïve patients $(n=19)$ and patients who had progressed on abiraterone or enzalutamide but continued $(n=10)$ or discontinued $(n=14)$ treatment. The box plots show the AR nuclear expression in white blood cells in the same sample. Samples were sorted first on more or equal to five aCTCs and then by median AR expression (only patients with $\geq 1 \mathrm{aCTC}$ were included). (B) Percentage of aCTCs divided by classes of AR intensity expression. Only patients with $\geq 4$ aCTCs were included. (C) Waterfall plot of the maximum percentage change in nuclear AR intensity of CTCs at baseline and at progression. Bars represent individual patients and grey colour correspond to response to treatment defined as a confirmed $>50 \%$ decline in PSA in the absence of radiological progression. Increases were capped at $100 \%$. (D) Scatter plot of nuclear AR intensity of individual cancer cells in formalin-fixed paraffin-embedded tissue CRPC tissue sections. Samples were sorted, first on previous exposure to abiraterone and second on the median intensity of AR. Median intensity \pm IQR is shown (n.c.-normalised counts). 

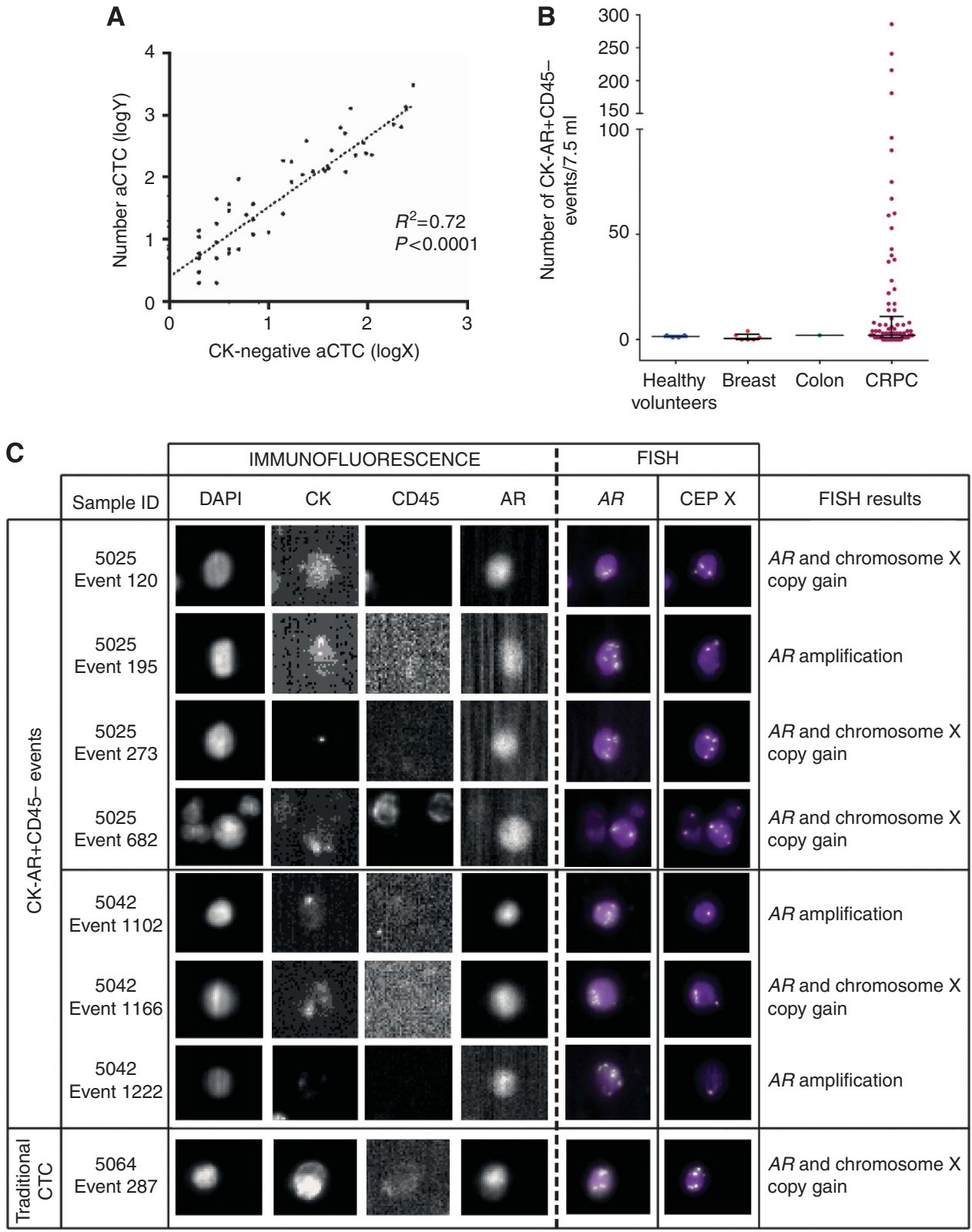

Figure 4. Detection of AR + CK-weak CTCs. (A) CK-AR + CD45- nucleated CTC counts significantly correlated with traditional CK + aCTC. Linear regression. (B) Number of CK-AR +CD45- events detected in male healthy volunteers $(N=4)$, breast cancer $(N=6)$, colon cancer $(N=1)$ and CRPC patients $(N=48)$. Median \pm IQR is shown. (C) AR amplification and copy number gain was detected by FISH on CK-AR + CD45- events confirming malignant origin of these cells. Internal controls of the FISH method can be seen on the sample 5025 (event 682 ) containing three leukocytes with one AR/chromosome X copy number per leukocyte. Last image shows an example of a traditional CK + CTC with AR and $X$ chromosome copy gain.

weak/negative cells was associated with overall survival in univariate Cox Regression analysis (continuous) $(P=0.004)$ in CRPC patients.

\section{DISCUSSION}

The mechanisms that underlie resistance to the novel AR targeting agents, abiraterone and enzalutamide, and how to overcome these are largely unknown, and their elucidation is a priority in prostate cancer research. A major question currently is whether the predominant mechanism mediating resistance is still through AR or whether AR-independent mechanisms are more common. In this study, we aimed to evaluate nuclear AR expression in CRPC patients treated with the AR-targeting agents abiraterone and enzalutamide. We developed an online protocol to determine in real-time nuclear AR expression in CTCs using the CellSearch system, and we employed an automated algorithm to identify CTCs and quantify AR expression. We compared patients grouped by absence of prior exposure $v s$ resistance to abiraterone or enzalutamide. We observed large intra- and interpatient heterogeneity of AR expression in CTCs, but we did not find a significant difference in nuclear AR expression in CTCs in resistant CRPC. Furthermore, we confirmed this conclusion in patient-matched CTC samples collected before starting treatment and at progression $(n=8)$, and in tumour biopsies from patients progressing on abiraterone $(n=13)$. We collected samples during the first cycle of treatment but owing to a rapid decline in CTC count in patients who responded to treatment, we were unable to demonstrate whether a decline in PSA was associated with 
reduced AR expression in CTCs because of insufficient CTCs captured for these evaluations.

Owing to the requirement to co-stain for CK, CD45 and DAPI to identify CTCs, we were limited to evaluation of only one additional marker on CellSearch. Our data suggest absence/low AR expression as a possible artefact of loss of cellular integrity despite cells remaining CK-positive. Previous studies have used markers of apoptosis such as M30 to identify the loss of viability in CTCs and demonstrate that this associated with failure of FISH to work (Larson et al, 2004; Swennenhuis et al, 2009). Our observations suggest that AR negativity in CTCs should be interpreted with caution. In addition, with this AR assay we identified circulating cells that are AR-positive but CK-negative/weak. In three patients, we were able to confirm the malignant origin of these circulating cells by $A R$ FISH. These events could either represent a biologically distinct sub-type associated with an epithelial-mesenchymal transition (Bitting et al, 2013) or traditional CTC with weaker CK staining because of a technical limitation or imaging artifact. Further studies are required to confirm the nature of these CK-weak/negative circulating cells. Critically, they would be missed using standard CTC criteria but are amenable to molecular characterisation.

Several mechanisms have been proposed that can explain our observation of maintained nuclear AR expression at progression on abiraterone or enzalutamide, including the presence of activating AR mutations or the expression of truncated AR splice variants (Attard et al, 2009a; Richards et al, 2012; Carreira et al, 2014). The antibody used in this study targets the amino-terminus of the AR and cannot therefore discriminate between full-length $\mathrm{AR}$ and truncated splice variants. The latter may cause resistance to drugs targeting the ligand-binding domain of the AR such as abiraterone and enzalutamide and explain AR nuclear localisation in patients progressing on these agents (Antonarakis et al, 2014). Up to this day, we are not aware of a sufficiently robust conjugated antibody for the detection of AR splice variants (e.g., ARV7, $\mathrm{AR}^{567 \mathrm{es}}$ ) that could be used on the CellSearch platform. However, the protocol we present could be adapted to study AR variant specific antibodies if they become available. In summary, we developed a non-invasive method to monitor AR nuclear expression in CRPC. Our studies suggest no change in nuclear AR expression following development of resistance to novel endocrine agents in CRPC. Owing to the significant heterogeneity of AR expression in CTCs, studies in larger cohorts of patients are required to identify associations with outcome.

\section{ACKNOWLEDGEMENTS}

This study was funded by the Movember CTC GAP programme, EU FP7 (Grant agreement no: 305341), Prostate Cancer UK, and the Prostate Cancer Foundation, Santa Monica, US. RF was supported by The Wellcome Trust (Clinical PhD Programme), DL was supported by the 'Beca SEOM para la Investigacion Traslacional' by the Spanish Oncology Society and GA was supported by a Cancer Research UK Clinician Scientist Fellowship. The authors acknowledge NHS funding to the RMH NIHR Biomedical Research Centre. We thank all the participating men and their families who suffered from metastatic prostate cancer and nonetheless gave the gift of participation so that others might benefit.

\section{CONFLICT OF INTEREST}

The ICR developed abiraterone and therefore has a commercial interest in this agent. GA is on the ICR list of rewards to inventors for abiraterone. JSdeB has received consulting fees and travel support from Amgen, Astellas, AstraZeneca, Boehringer Ingelheim, Bristol-Myers Squibb, Dendreon, Enzon, Exelixis, Genentech, GlaxoSmithKline, Medivation, Merck, Novartis, Pfizer, Roche, Sanofi-Aventis, Supergen, and Takeda and grant support from AstraZeneca and Genentech. GA has received honoraria, consulting fees or travel support from Astellas, Medivation, Janssen, Millennium Pharmaceuticals, Ipsen, Takeda, and Sanofi-Aventis and grant support from Janssen and AstraZeneca. LWMMT receives grant support from Janssen Diagnostics.

\section{REFERENCES}

Allard WJ, Matera J, Miller MC, Repollet M, Connelly MC, Rao C, Tibbe AG, Uhr JW, Terstappen LW (2004) Tumor cells circulate in the peripheral

blood of all major carcinomas but not in healthy subjects or patients with nonmalignant diseases. Clin Cancer Res 10(20): 6897-6904.

Antonarakis ES, Lu C, Wang H, Luber B, Nakazawa M, Roeser JC, Chen Y, Mohammad TA, Chen Y, Fedor HL, Lotan TL, Zheng Q, De Marzo AM, Isaacs JT, Isaacs WB, Nadal R, Paller CJ, Denmeade SR, Carducci MA, Eisenberger MA, Luo J (2014) AR-V7 and resistance to enzalutamide and abiraterone in prostate cancer. N Engl J Med 371(11): 1028-1038.

Arora VK, Schenkein E, Murali R, Subudhi SK, Wongvipat J, Balbas MD, Shah N, Cai L, Efstathiou E, Logothetis C, Zheng D, Sawyers CL (2013) Glucocorticoid receptor confers resistance to antiandrogens by bypassing androgen receptor blockade. Cell 155(6): 1309-1322.

Attard G, Cooper CS, de Bono JS (2009a) Steroid hormone receptors in prostate cancer: a hard habit to break? Cancer Cell 16(6): 458-462.

Attard G, de Bono JS (2011) Utilizing circulating tumor cells: challenges and pitfalls. Curr Opin Genet Dev 21(1): 50-58.

Attard G, Swennenhuis JF, Olmos D, Reid AH, Vickers E, A'Hern R, Levink R, Coumans F, Moreira J, Riisnaes R, Oommen NB, Hawche G, Jameson C, Thompson E, Sipkema R, Carden CP, Parker C, Dearnaley D, Kaye SB, Cooper CS, Molina A, Cox ME, Terstappen LW, de Bono JS (2009b) Characterization of ERG, AR and PTEN gene status in circulating tumor cells from patients with castration-resistant prostate cancer. Cancer Res 69(7): 2912-2918.

Beltran H, Tagawa ST, Park K, MacDonald T, Milowsky MI, Mosquera JM, Rubin MA, Nanus DM (2012) Challenges in recognizing treatment-related neuroendocrine prostate cancer. J Clin Oncol 30(36): e386-e389.

Bianchini D, Lorente D, Rodriguez-Vida A, Omlin A, Pezaro C, Ferraldeschi R, Zivi A, Attard G, Chowdhury S, de Bono JS (2014) Antitumour activity of enzalutamide (MDV3100) in patients with metastatic castration-resistant prostate cancer (CRPC) pre-treated with docetaxel and abiraterone. Eur J Cancer 50(1): 78-84.

Bitting RL, Boominathan R, Rao C, Kemeny G, Foulk B, Garcia-Blanco MA, Connelly M, Armstrong AJ (2013) Development of a method to isolate circulating tumor cells using mesenchymal-based capture. Methods 64(2): 129-136.

Carreira S, Romanel A, Goodall J, Grist E, Ferraldeschi R, Miranda S, Prandi D, Lorente D, Frenel JS, Pezaro C, Omlin A, Rodrigues DN, Flohr P, Tunariu N, SdB J, Demichelis F, Attard G (2014) Tumor clone dynamics in lethal prostate cancer. Sci Transl Med 6(254): 254ra125.

Darshan MS, Loftus MS, Thadani-Mulero M, Levy BP, Escuin D, Zhou XK, Gjyrezi A, Chanel-Vos C, Shen R, Tagawa ST, Bander NH, Nanus DM, Giannakakou P (2011) Taxane-induced blockade to nuclear accumulation of the androgen receptor predicts clinical responses in metastatic prostate cancer. Cancer Res 71(18): 6019-6029.

de Bono JS, Attard G, Adjei A, Pollak MN, Fong PC, Haluska P, Roberts L, Melvin C, Repollet M, Chianese D, Connely M, Terstappen LW, Gualberto A (2007) Potential applications for circulating tumor cells expressing the insulin-like growth factor-I receptor. Clin Cancer Res 13(12): 3611-3616.

de Bono JS, Logothetis CJ, Molina A, Fizazi K, North S, Chu L, Chi KN, Jones RJ, Goodman Jr OB, Saad F, Staffurth JN, Mainwaring P, Harland S, Flaig TW, Hutson TE, Cheng T, Patterson H, Hainsworth JD, Ryan CJ, Sternberg CN, Ellard SL, Flechon A, Saleh M, Scholz M, Efstathiou E, Zivi A, Bianchini D, Loriot Y, Chieffo N, Kheoh T, Haqq CM, Scher HI (2011) Abiraterone and increased survival in metastatic prostate cancer. N Engl J Med 364(21): 1995-2005. 
Larson CJ, Moreno JG, Pienta KJ, Gross S, Repollet M, O'Hara SM, Russell T, Terstappen LW (2004) Apoptosis of circulating tumor cells in prostate cancer patients. Cytometry A 62(1): 46-53.

Ligthart ST, Bidard FC, Decraene C, Bachelot T, Delaloge S, Brain E, Campone M, Viens P, Pierga JY, Terstappen LW (2013) Unbiased quantitative assessment of Her-2 expression of circulating tumor cells in patients with metastatic and non-metastatic breast cancer. Ann Oncol 24(5): 1231-1238.

Ligthart ST, Coumans FAW, Attard G, Mulick Cassidy A, de Bono JS, Terstappen LWMM (2011) Unbiased and automated identification of a circulating tumour cell definition that associates with overall survival. Plos One 6(11): e27419.

Liu Y, Liu Q, Wang T, Bian L, Zhang S, Hu H, Li S, Hu Z, Wu S, Liu B, Jiang Z (2013) Circulating tumor cells in HER2-positive metastatic breast cancer patients: a valuable prognostic and predictive biomarker. BMC Cancer 13: 202.

Loriot Y, Bianchini D, Ileana E, Sandhu S, Patrikidou A, Pezaro C, Albiges L, Attard G, Fizazi K, De Bono JS, Massard C (2013) Antitumour activity of abiraterone acetate against metastatic castration-resistant prostate cancer progressing after docetaxel and enzalutamide (MDV3100). Ann Oncol 24(7): 1807-1812.

Malvezzi M, Bertuccio P, Levi F, La Vecchia C, Negri E (2014) European cancer mortality predictions for the year 2014. Ann Oncol 25(8): $1650-1656$.

Miyamoto DT, Lee RJ, Stott SL, Ting DT, Wittner BS, Ulman M, Smas ME, Lord JB, Brannigan BW, Trautwein J, Bander NH, Wu CL, Sequist LV, Smith MR, Ramaswamy S, Toner M, Maheswaran S, Haber DA (2012) Androgen receptor signaling in circulating tumor cells as a marker of hormonally responsive prostate cancer. Cancer Discov 2(11): 995-1003.

Punnoose EA, Atwal S, Liu W, Raja R, Fine BM, Hughes BG, Hicks RJ, Hampton GM, Amler LC, Pirzkall A, Lackner MR (2012) Evaluation of circulating tumor cells and circulating tumor DNA in non-small cell lung cancer: association with clinical endpoints in a phase II clinical trial of pertuzumab and erlotinib. Clin Cancer Res 18(8): 2391-2401.
Richards J, Lim AC, Hay CW, Taylor AE, Wingate A, Nowakowska K, Pezaro C, Carreira S, Goodall J, Arlt W, McEwan IJ, de Bono JS, Attard G (2012) Interactions of abiraterone, eplerenone, and prednisolone with wild-type and mutant androgen receptor: a rationale for increasing abiraterone exposure or combining with MDV3100. Cancer Res 72(9): 2176-2182.

Sahu B, Laakso M, Pihlajamaa P, Ovaska K, Sinielnikov I, Hautaniemi S, Janne OA (2013) FoxA1 specifies unique androgen and glucocorticoid receptor binding events in prostate cancer cells. Cancer Res 73(5): 1570-1580.

Scher HI, Fizazi K, Saad F, Taplin ME, Sternberg CN, Miller K, de Wit R, Mulders P, Chi KN, Shore ND, Armstrong AJ, Flaig TW, Flechon A, Mainwaring P, Fleming M, Hainsworth JD, Hirmand M, Selby B, Seely L, de Bono JS (2012) Increased survival with enzalutamide in prostate cancer after chemotherapy. N Engl J Med 367(13): 1187-1197.

Scher HI, Halabi S, Tannock I, Morris M, Sternberg CN, Carducci MA, Eisenberger MA, Higano C, Bubley GJ, Dreicer R, Petrylak D, Kantoff P, Basch E, Kelly WK, Figg WD, Small EJ, Beer TM, Wilding G, Martin A, Hussain M. Prostate Cancer Clinical Trials Working G (2008) Design and end points of clinical trials for patients with progressive prostate cancer and castrate levels of testosterone: recommendations of the Prostate Cancer Clinical Trials Working Group. J Clin Oncol 26(7): 1148-1159.

Swennenhuis JF, Tibbe AG, Levink R, Sipkema RC, Terstappen LW (2009) Characterization of circulating tumor cells by fluorescence in situ hybridization. Cytometry A 75(6): 520-527.

Wang LH, Pfister TD, Parchment RE, Kummar S, Rubinstein L, Evrard YA, Gutierrez ME, Murgo AJ, Tomaszewski JE, Doroshow JH, Kinders RJ (2010) Monitoring drug-induced gammaH2AX as a pharmacodynamic biomarker in individual circulating tumor cells. Clin Cancer Res 16(3): 1073-1084.

This work is published under the standard license to publish agreement. After 12 months the work will become freely available and the license terms will switch to a Creative Commons AttributionNonCommercial-Share Alike 4.0 Unported License.

Supplementary Information accompanies this paper on British Journal of Cancer website (http://www.nature.com/bjc) 